\title{
Analysis of Nonlinear Characteristics of Turbine Governor and Its Impact on Power System Oscillation
}

\author{
Ge Jin ${ }^{1}$, Hanting Yan ${ }^{2}$, Shaoxiang Deng ${ }^{1}$, Xiaomei Chen ${ }^{2}$, Zexiang Cai ${ }^{2}$ \\ ${ }^{1}$ Electric Power Research Institute, Guangdong Power Grid, Guangzhou, China \\ ${ }^{2}$ School of Electric Power, South China University of Technology, Guangzhou, China \\ Email: yanhanting@126.com
}

Received March, 2013

\begin{abstract}
In this paper, the model of turbine governor based on the physical principles is constituted to improve the accuracy of power system dynamic simulation, making the results of simulation consistent with the actual situation. The unit and grid coupling model which reflects the interaction between thermal system of power plant and power system is built using EMTDC/PSCAD. The influence of nonlinear characteristics on the valve opening and the steam turbine mechanical power is also analyzed in this paper. The results of simulation show that the improper setting of parameters reflecting nonlinear characteristics of turbine governor can lead to the cyclical oscillations of the valve opening and the steam turbine mechanical power, and even can lead to the power oscillation persistently. The research achievements in this paper have a certain reference value on exploring the causes of power oscillation from the prime mover.
\end{abstract}

Keywords: Turbine Governor; Modeling; Nonlinear Characteristics; Power System Oscillation

\section{Introduction}

In the analysis of power system stability, the response speed of traditional governor is slow and the time constant is large, the mechanical power of prime mover can be approximately considered as a constant, so the dynamic characteristics of governor and prime mover can be ignored. However, with the rapid development of power system, the response speed of governor has been improved greatly. So, when the power system is under disturbance, it is necessary to consider the response characteristics of the governor [1]. The power system dynamic process of the unit and grid coordination is very complicated. The governor model of prime mover for dynamic simulation is very different in different time domain, but the current governor models are simplified, which results in the decrease of simulation precision, and a greater difference between the simulation results and the accident records. Therefore, the modeling of governor specific to different dynamic process is critical to the power system dynamic simulation.

Steam turbine governor system mostly adopts the digital electro-hydraulic control system (DEH), which is a dynamic system composed of many elements. It occupies a very important position in the turbo-generator unit. Therefore, building complete mathematical model of the turbine governor, understanding its working mechanism and analyzing its influence on the power system dynamic simulation are a very meaningful research subject. In reference [2], a conclusion is drawn through the simulation that if the speed variation ratio is locally too small, a power system low frequency oscillation with resonance mechanism may occur. In reference [3], improper selection of the governor parameter will provide negative damping to exacerbate low frequency oscillation of the system.

This paper will be organized as follows. First, the characteristics of the digital electro-hydraulic control system (DEH) are analyzed deeply. Secondly, the model of turbine governor considering the nonlinear characteristics is constituted. Last, the research of the nonlinear characteristics and their impacts on the power system are performed. Research shows that the nonlinear characteristics of the governor have a certain influence on the power system oscillation.

\section{Modeling of Turbine Governor Considering Nonlinear Characteristics}

At present, in the power system dynamic simulation, governor models are simplified ignoring the nonlinear characteristics of the governor, which results in the decrease of simulation precision, and a greater difference between the simulation results and the accident records. However, in fact, because of factors such as machinery manufacturing, the governor has some nonlinear characteristics, such as the rate-limit characteristic, amplitude-limit characteristic, dead band characteristic, clear- 
ance nonlinearity characteristic, etc [4]. These nonlinear characteristics may lead to the cyclical fluctuation of regulating valve, which will cause cyclical fluctuation of the steam turbine mechanical power.

DEH system is composed of pure electric and hydraulic servo system, with the digital computer as the controller of the DEH system, the electro-hydraulic conversion mechanism and the oil motor as its actuator. It is the ideal governor system of steam turbine at present [5]. The DEH system usually has speed control, load control, over-speed protection, steam turbine generator parameters monitoring, as well as on-off sequence control function. The modeling of DEH governor can be divided into two parts, the modeling of the control part and the modeling of the actuator.

The control part of DEH has 3 kinds, namely the valve position control, load control and the regulating pressure control. Actuator adjusts the valve to the specified location according to the turbine control valve instructions received from the control part. Actuator itself is a closed loop system. It accepts instruction from the governor system, and then forms control signals according to the deviation of current opening and the received instruction, some add the PID control law in order to achieve the rapidity and accuracy requirements. Electro-hydraulic converter is one of the pivotal components in the digital electro-hydraulic control system (DEH). Electro-hydraulic converter connects the part of electric with the hydraulic actuator, and at the same time, enlarge weak electrical signal into hydraulic signal. Finally, the hydraulic actuator with strong power controls the regulating valve, achieving automatic adjustment. The hydraulic actuator is composed of relay, wrong throttle and oil motor. Due to friction, clearance, the insensitive area of the detecting element, machinery manufacturing and other factors, the governor has some nonlinear characteristic [6].

Steam turbine governor system generally adopts the slide valve oil motor as its actuator. In order to reduce the interference that pulse pressure fluctuation bring to the oil motor, slide valve need to have a certain overlapping degree, which cause the slide valve is not sensitive. At the same time, the slide valve overcomes the dry friction during sliding movement. The friction force is always in the opposite direction with the valve movement, which also leads to the insensitive of governing system. In practice, because of the overlapping degree and the dry friction of slide valve, the turbine governor has the dead band characteristic. In addition, the load of electric power system is random, so the frequency of power system has natural fluctuation characteristic. The fluctuation range is about $0.2 \mathrm{~Hz}$, in which governor system is not expected to work, lest the frequency fluctuation of power system would increase. When the frequency of Power system changes, the unit with electro-hydraulic regulating system would grab the load due to the high sensitivity, and the unit acted as basic load is not expected to participate in the load distribution, and the load is not expected to change frequently while rate changes [7].Therefore, in the power system simulation, in order to stabilize the load, reduce the frequency of adjustment, the governor has the dead band characteristic.

Hydraulic actuator has a certain rate limit when controls the movement of regulating valve. If the rate limit is near to the setting value of regulating valve, the regulating rate of valve would not be proportional to the rate of the linear system [8]. Therefore, the turbine governor has the rate-limit characteristic.

The turbine governor has the amplitude-limit characteristic, when the flow of slide valve or the displacement of oil motor reaches the boundary. In the case of small disturbance, the system performs in the linear area of amplitude-limit characteristic of the slide valve and the oil motor. In the case of great disturbance (such as load shedding, etc.), the flow of slide valve and the position of oil motor reach the limit, so, the nonlinear area of amplitude-limit characteristic works, limiting the response amplitude of system [9]. As a result, it can prevent overload, reduce the gain of the system and improve the stability of the system.

Because of the slip ring of the governor need to overcome factors such as the friction, clearance, and the overlapping degree of wrong throttle when moving. The shift of oil motor piston forms the hysteresis; as a result, the turbine governor has the clearance nonlinear characteristic [10].

To sum up, the model of the turbine governor considering nonlinear characteristics can be built, as shown in Figure 1.
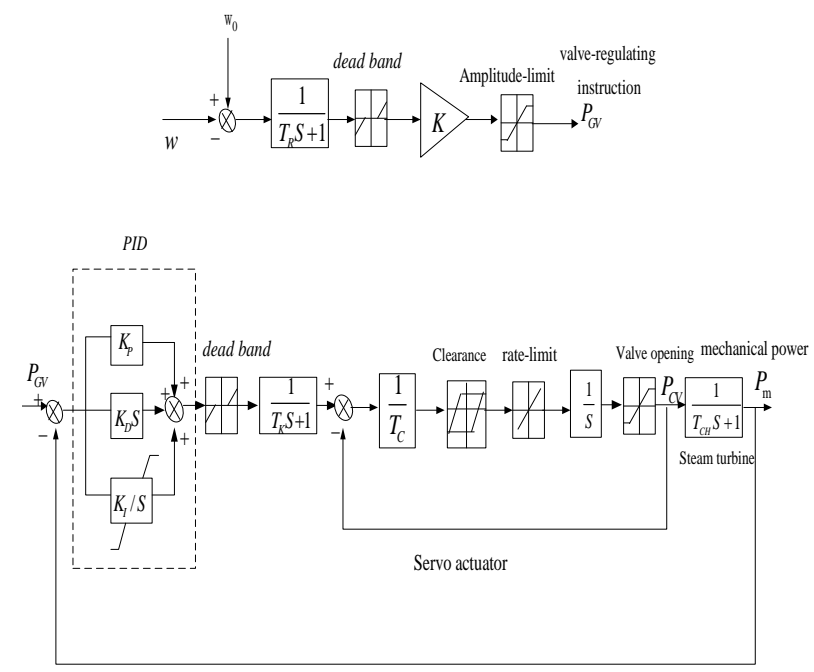

Figure 1. The model of turbine governor considering nonlinear characteristics. 


\section{Analysis of the Influence of Nonlinear Characteristics}

\subsection{The Influence of Dead Band Characteristic}

The setting of the dead band has a greater impact on the primary frequency regulation of the unit. When the frequency of power system fluctuates in a small area, the dead band is to prevent the unsafe operation of equipments caused by unnecessary working of the turbine control valve frequently. To analyze the influence of dead band, this paper take no account of the rate-limit characteristic, amplitude-limit characteristic, clearance nonlinear characteristic, and adjust the value of dead band under $0.1 \mathrm{pu}$ external load disturbance. When the setting value of dead band is less than $0.057 \mathrm{~Hz}$, valve opening degree and mechanical power have no obvious fluctuation. When the setting value of dead zone is more than $0.057 \mathrm{~Hz}$, valve opening degree and mechanical power have obvious fluctuation, as shown in Figure 2.

\subsection{The Influence of Rate-limit Characteristic}

The typical parameters reflecting the rate-limit characteristic of the turbine digital electro-hydraulic control system are as follows: upper to 1.0 , lower to -3.0 , which requires the rise rate (the turning up rate of valve adjustment) of the governor less than 1.0. The upper limit is looser. If the rate limit is near to the setting value of regulating valve, the regulating rate of valve would not be proportional to rate of the linear system. Therefore, it is not suitable for the analysis of real system. To analyze the influence of rate-limit characteristic, this paper take no account of the dead band characteristic, amplitudelimit characteristic, clearance nonlinear characteristic, and adjust the value of rate limit under $0.1 \mathrm{pu}$ external load disturbance, making it close to the rate of actual valve adjustment slowly.

When the upper limit decreases to 0.29 , we can find that the steam valve fluctuates obviously, and the amplitude increases slowly. When the upper limit is 0.28 , the wave of steam valve oscillates persistently, as shown in Figure 3. At this moment, the upper limit reflecting the nonlinear rate-limit characteristic is below the actual adjustment rate of the valve. To sum up, if the upper limit reflecting the nonlinear rate-limit characteristic of the governor is set inappropriately, making it near to the actual regulating rate of the adjusting valve, it may lead to the cyclical fluctuation of valve opening degree and the steam turbine mechanical power.

\subsection{The Influence of Amplitude-limit Characteristic}

The amplitude limit of the general thermal power generating unit is $\pm 6 \%- \pm 10 \%$. To analyze the influence of
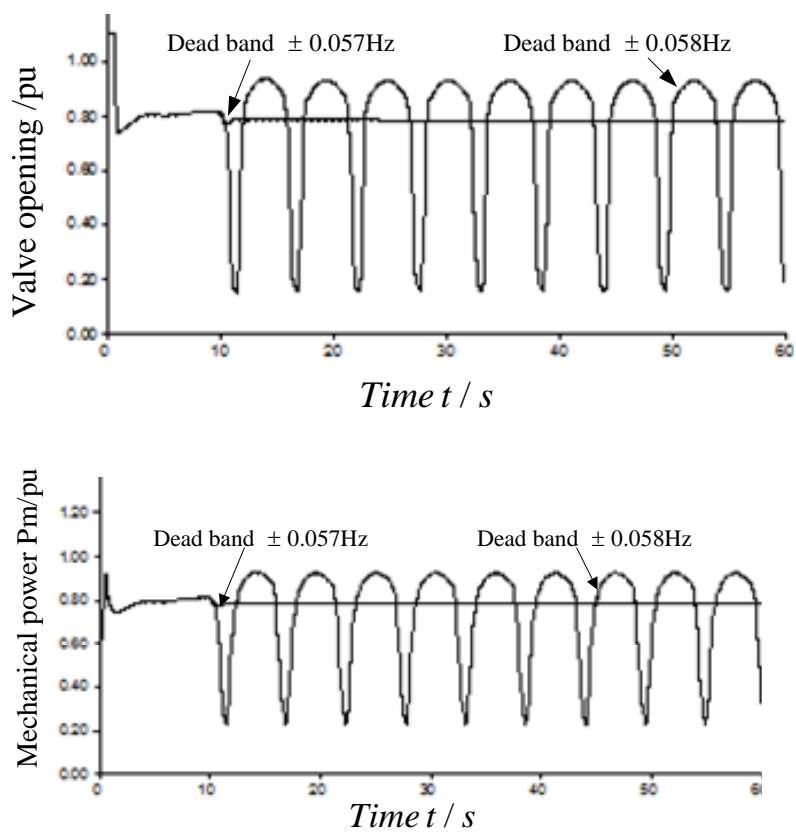

Figure 2. Valve opening degree and mechanical power under different value of dead band.
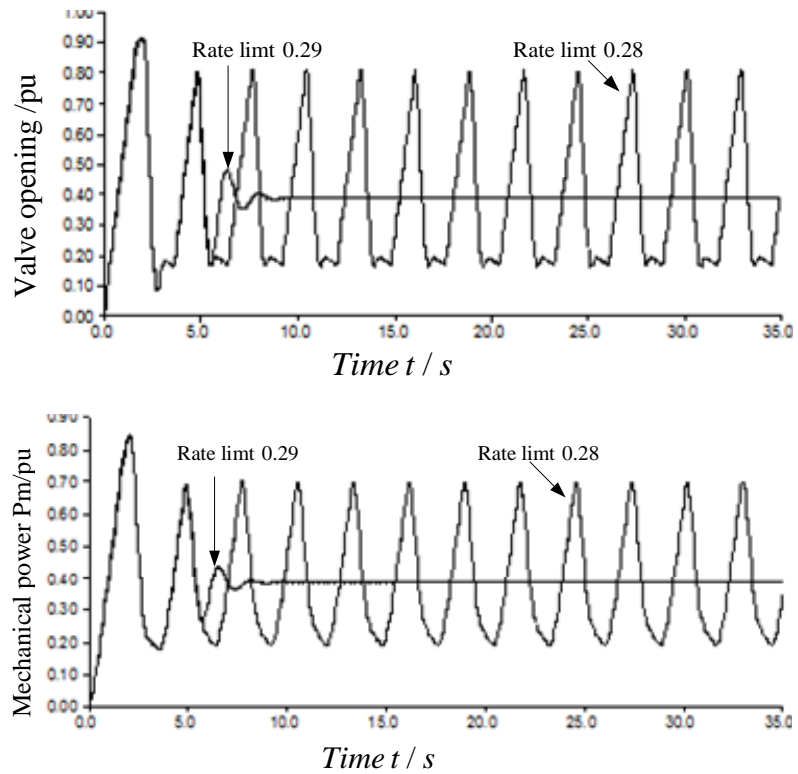

Figure 3. Valve opening degree and mechanical power under different value of rate limit.

amplitude-limit characteristic, this paper take no account of the dead band characteristic, rate-limit characteristic, clearance nonlinear clearance, and adjust the value of amplitude limit under $0.1 \mathrm{pu}$ external load disturbance (operating value set as 106\%). When the upper and lower limits decrease to $\pm 5 \%$, the valve opening degree and mechanical power have no obvious fluctuation. When the upper and lower limits decrease to $\pm 4 \%$, the valve opening degree and mechanical power have obvious fluctua- 
tion, as shown in Figure 4.

\subsection{The Influence of Clearance Nonlinearity Characteristic}

In the electro-hydraulic servo system, the clearance nonlinearity is mainly produced by torque motor. This kind of nonlinear characteristic mainly affects the sensitivity and the static error of system. The effect on the dynamic characteristics is a fixed phase shift. It has no serious influence on the stability of the system. The graph and mathematical expressions of clearance nonlinearity characteristic are shown in Figure 5.


Figure 4. Valve opening degree and mechanical power under different amplitude limit.

$$
\bar{u}(k)=\left\{\begin{array}{l}
\text { Ku(k) } \\
K u(k)+R, \begin{array}{l}
\text { 当 } u(k)>u(k-1) \\
\text { 当 } u(k) \geq u(k)<u(k-1) \\
\text { 当 } u(k-1), \text { others }
\end{array}
\end{array}\right.
$$

Figure 5. The graph and mathematical expressions of clearance nonlinear.

\section{The Impact of Nonlinear Characteristics of Turbine Governor on Power System Oscillation}

The power system unit and grid simulation environment is established in EMTDC/PSCAD, including the steam turbine, the governor considering the nonlinear characteristics, the generator, the excitation and the load model, as shown in Figure 6. With the single machine infinite system as an example, this paper analyzes the impact of nonlinear characteristics of turbine governor on the power system oscillation.

When the nonlinear characteristics of the governor system give rise to the cyclical fluctuations of the regulating valve, the mechanical power of the unit may fluctuate cyclically. What is more, the active power of transmission line may also engender cyclically oscillation.

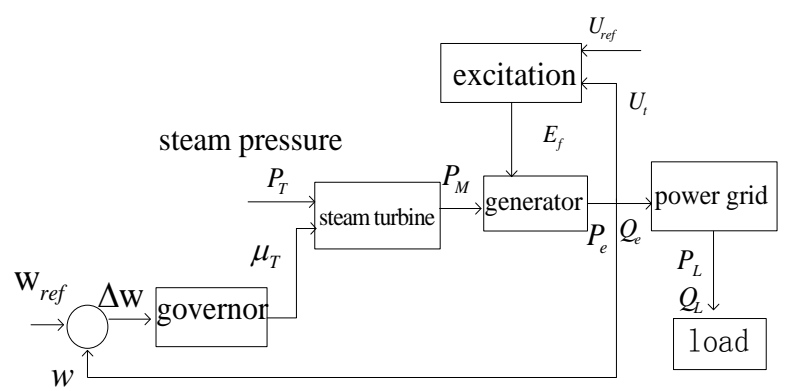

Figure 6. Structure diagram of machine-grid coupling model.
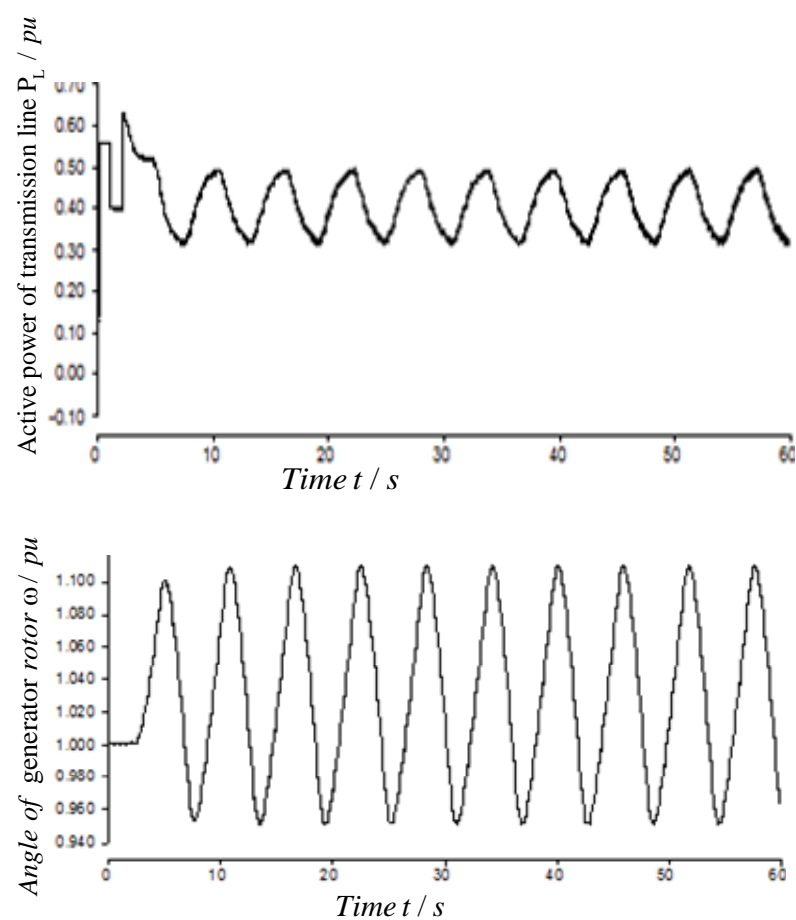

Figure 7. Active power of transmission line and the angle of generator rotor. 
Taking the dead band characteristic as an example, when the value of dead band is greater than $0.057 \mathrm{~Hz}$, the valve opening degree and mechanical power will have obvious fluctuations, meanwhile, the active power of transmission line and the angle of generator rotor also have cyclically oscillations, as shown in Figure 7.

\section{Conclusions}

The nonlinear characteristics of turbine governor actually exist in the real system, but the governor model of BPA and other simulation software does not take into account the nonlinear characteristics, leading to the simulation results inconsistent with the actual situation in some cases. Therefore, the modeling of turbine governor considering the nonlinear characteristics is very necessary. The nonlinear characteristics have a certain influence on the stability of the system, the parameters reflecting the dead band characteristic, the rate-limit characteristics and the amplitude-limit characteristics set improperly can cause the regulating valve degree and the mechanical power to fluctuate cyclically, and then give rise to the power oscillations of transmission line.

\section{REFERENCES}

[1] D. R. Yu and J. Y.Xu, "The Effects of Governor On The Stability of Turbo-Generator," Power System Automation, Vol. 20, No. 1,1996, pp.23-26.

[2] Z. Y. Han, Y. H. Xu, Y. Li and C. Q. Wu, "Power System
Low Frequency Oscillation of Resonance Mechanism Induced by Disturbance of Turbine-Governing System," Shaanxi Electric Power, Vol. 7, 2009, pp. 1-5.

[3] G. H. Wang and X. Huang, "Influence of Turbine Governor Parameters on Power System Damping," Electric Power Automation Equipment, Vol. 31, No. 4, 2009, pp. 87-90.

[4] Y. Wang, Y. S. Wang and Z. H. Li, "Model and Nonlinear Analysis of 300MW Steam Turbine Speed Control System,” Steam Turbine Technology, Vol. 49, No. 1, 2007, pp. 17-20.

[5] G. X. Miao, "Parameter Identification of Steam Turbine Digital Electro-hydraulic Control System and Application," Baoding: north China electric power university, 2004.

[6] H. Q. Lv, Y. Su, J. Y. Yang and Z. X. Li, “The Nonlinear Characteristics of Electro-hydraulic Servo System and Its Effect Analysis,” Machine Tool and Hydraulics, 2002.

[7] D. R. Yu, W. Bo and W. T. Ju, "The Effects of Nonlinear Characteristics of Steam Turbine Governor System on The Torsional Stability," Steam Turbine Technology, Vol. 33, No. 5, 1997, pp. 257-261.

[8] C. D. Vournas, "Unstable Frequency Oscillation in a Slow-Response Reheat-Turbine Generator," IEEE Transactions on Automatic Control, 1998.

[9] Z. P. Zhou, "First Frequency Modulation Delimitation for Electric System Stabilization Analyze,” Henan Electric Power, Vol. 3, 2009, pp. 40-45.

[10] Y. S. Hao, L. N. Zeng and Y. H. Yang, "Analysis of The Influence of Governor Back-lashon GPSS,” North China Electric Power University Acta, Vol. 4, 1992, pp. 1-6. 